
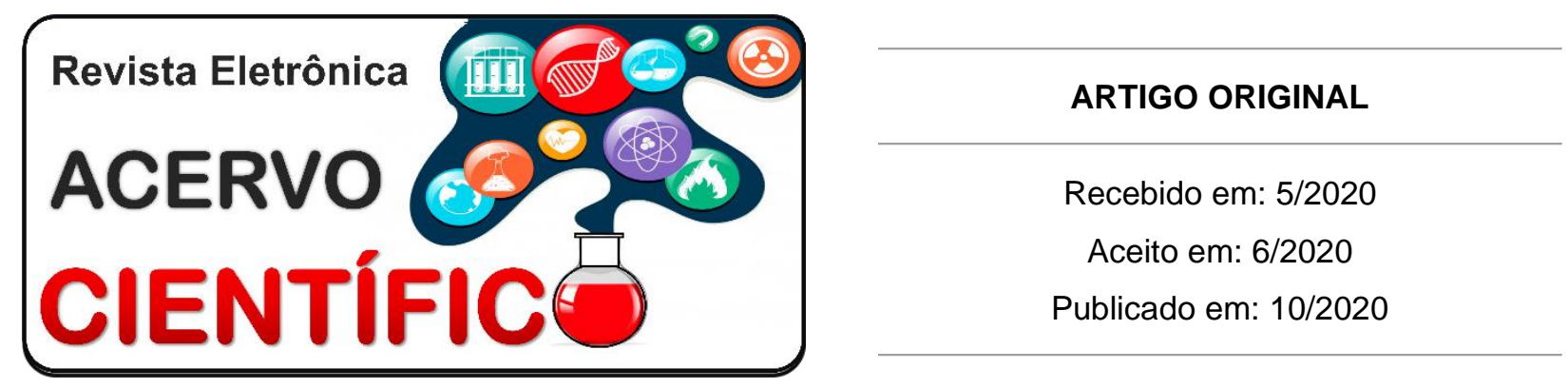

\title{
Tuberculose pulmonar: prevalência em um território de abrangência de uma unidade de saúde da família em Belém
}

\author{
Pulmonary tuberculosis: prevalence in a territory of scope of a family health unit in Belem \\ Tuberculosis pulmonar: prevalencia en un territorio de una unidad de salud familiar en \\ Belém
}

Gabriela Rosa Bernardo ${ }^{1 *}$, Fernanda Moema Mendes Leite ${ }^{1}$, Fernanda Vidal Pacheco ${ }^{1}$, Karen Regina Guimarães Dantas ${ }^{1}$, Mariana Rosa Bernardo ${ }^{1}$, Afonso Vinicius de Lima Filgueira ${ }^{1}$, Bruno da Silva Quaresma ${ }^{1}$, Rafael Schalcher Martins e Silva ${ }^{2}$, Renata Ogawa Furtado Rodrigues ${ }^{1}$, Osvaldo da Silva Peixoto'.

\begin{abstract}
Resumo: Identificar a taxa de prevalência de tuberculose pulmonar no território de uma Unidade de Saúde da Família do Canal de uma cidade do Pará. Estudo quantitativo, transversal, descritivo realizada com os indivíduos com tuberculose cadastrados na Estratégia Saúde da Família em uma cidade do Pará. A coleta de informações ocorreu por meio da análise de prontuários e aplicação de questionário, nos quais foram utilizados dados. Foram avaliados 39 participantes, sendo 56\% mulheres, 54\% pardos, $44 \%$ com ensino fundamental incompleto, $72 \%$ realizam atividade remunerada, sendo $68 \%$ autônomo, $72 \%$ tem autocontribuição com a renda familiar, $91 \%$ recebe menos de um salário mínimo, 59\% tem cinco ou mais dependentes, $32 \%$ não tem jornada fixa, $49 \%$ mora em casa cedida, $39 \%$ tem quatro cômodos na casa, $31 \%$ reside com mais três pessoas, $67 \%$ tem casa de alvenaria, $62 \%$ com telhado de telhas de barro, $82 \%$ não tem boa ventilação na casa, $100 \%$ com banheiro no domicílio, sendo $92 \%$ dentro, $100 \%$ com água canalizada, 53\% com coleta regular de lixo, $67 \%$ sem doenças crônicas, sendo que dos que apresentaram $46 \%$ foi HIV. A associação entre tuberculose e indicadores socioeconômicos parece ser influenciada tanto pelo nível de agregação espacial quanto pelas características particulares das áreas geográficas.
\end{abstract}

Palavras-chave: Tuberculose, Epidemiologia, Atenção básica à saúde.

Abstract: Identify the prevalence rate of pulmonary tuberculosis in the territory of a Family Health Unit in the Canal of a city in Pará. Quantitative, cross-sectional, descriptive study carried out with individuals with tuberculosis registered in the Family Health Strategy in a city in Pará. Information collection occurred through the analysis of medical records and the application of a questionnaire, in which data were used. 39 participants were evaluated, of which $56 \%$ were women, $54 \%$ were brown, $44 \%$ had incomplete primary education, $72 \%$ performed paid work, $68 \%$ were self-employed, $72 \%$ had self-contribution with family income, $91 \%$ received less than one salary minimum, $59 \%$ have five or more dependents, $32 \%$ have no fixed hours, $49 \%$ live in a ceded house, $39 \%$ have four rooms in the house, $31 \%$ live with three more people, $67 \%$ have a brick house, $62 \%$ with clay tile roof, $82 \%$ do not have good ventilation in the house, $100 \%$ with a

${ }^{1}$ Centro Universitário Metropolitano da Amazônia (UNIFAMAZ), Belém - PA.

*E-mail: gabriela.trabalho@hotmail.com

${ }^{2}$ Centro Universitário do Planalto Central Apparecido dos Santos (UNICEPLAC), Brasília - DF. 
bathroom at home, $92 \%$ inside, $100 \%$ with running water, $53 \%$ with regular garbage collection, $67 \%$ without chronic diseases, of which that presented $46 \%$ was HIV. The association between tuberculosis and socioeconomic indicators seems to be influenced both by the level of spatial aggregation and by the particular characteristics of the geographical areas.

Keywords: Tuberculosis, Epidemiology, Primmary health care.

Resumen: Identificar la tasa de prevalencia de tuberculosis pulmonar en el territorio de una Unidad de Salud de la Familia en el Canal de una ciudad de Pará Estudio cuantitativo, transversal, descriptivo realizado con individuos con tuberculosis registrados en la Estrategia de Salud de la Familia en una ciudad de Pará. La recopilación de información se produjo mediante el análisis de registros médicos y la aplicación de un cuestionario, en el que se utilizaron los datos. 39 participantes fueron evaluados, $56 \%$ mujeres, $54 \%$ marrones, $44 \%$ con educación primaria incompleta, $72 \%$ realizan trabajo remunerado, $68 \%$ trabajan por cuenta propia, $72 \%$ tienen una contribución propia con ingresos familiares, $91 \%$ reciben menos de un salario mínimo, 59\% tiene cinco o más dependientes, $32 \%$ no tiene horas fijas, $49 \%$ vive en una casa cedida, $39 \%$ tiene cuatro habitaciones en la casa, 31\% vive con tres personas más, $67 \%$ tiene una casa de ladrillo, $62 \%$ con techo de tejas de arcilla, $82 \%$ no tiene buena ventilación en la casa, $100 \%$ con baño en casa, $92 \%$ adentro, $100 \%$ con agua corriente, 53\% con recolección regular de basura, $67 \%$ sin enfermedades crónicas, de las cuales que presentó $46 \%$ era VIH. La asociación entre la tuberculosis y los indicadores socioeconómicos parece estar influenciada tanto por el nivel de agregación espacial como por las características particulares de las áreas geográficas.

Palabras clave: Tuberculosis, Epidemiología, Atención primaria de salud.

\section{INTRODUÇÃO}

A tuberculose (TB) é uma doença infecciosa causada pela bactéria Mycobacterium tuberculosis e transmissível que afeta prioritariamente os pulmões causando tosse, febre, perda de peso e comprometimento da respiração. A doença é curável e o tratamento é feito com medicações disponibilizadas no Sistema Único de Saúde sendo uma doença de prognóstico bom se diagnosticado precocemente e tratado corretamente (VENDRAMINI SHF, et al., 2003).

O continente americano, Brasil e Peru contribuem com $50 \%$ dos casos estimados. Somando-se Bolívia, Equador, Haiti, Honduras, Guiana, México, Nicarágua e República Dominicana, chega-se a $75 \%$ dos casos estimados. Cabe salientar que no Brasil não é diferente, a tuberculose ainda é um sério problema para a saúde pública, com principais causas sociais. Outros 15 mil casos de re-tratamento, por recidiva ou reingresso após abandono, são notificados, a cada ano (BRASIL, 2016)

O Brasil está entre os 22 países do grupo de alta carga priorizados pela Organização Mundial da Saúde (OMS), que concentram $80 \%$ dos casos de tuberculose no mundo, ocupando a $16^{\text {a }}$ posição em casos apresentados. Em 2013, segundo o DATASUS, foram notificados 86108 casos, em 2014 - 85033 e em 2015 - 84397 em todo país (BRASIL, 2016)

No estado do Pará, a incidência de Tuberculose está decaindo no decorrer dos anos, porém ele ainda está entre um dos estados brasileiros que possuem os mais altos índices doença, devido, principalmente, ao fator socioeconômico. O boletim técnico da Secretaria de Estado de Saúde Pública- SESPA esclarece que a tuberculose é a primeira causa de morte entre as doenças infecciosas nos pacientes com Síndrome da Imunodeficiência Adquirida (AIDS) e a segunda de adoecimento (BRASIL, 2016).

Neste contexto, o Ministério da Saúde implantou medidas de acompanhamento, desenvolvidas com os objetivos principais de permitir o monitoramento dos pacientes atendidos e cadastrados na rede ambulatorial do Sistema Único de Saúde (SUS) e gerar informações para aquisição, dispensação e distribuição de medicamentos, de maneira sistemática, a esses pacientes (BRASIL, 2016). 
Hoje em dia, a Estratégia de Saúde da Família (ESF) proporciona acompanhamento aos portadores de TB, utilizando práticas que visam estabelecer novas relações entre profissionais de saúde, indivíduos, suas famílias e comunidade. A ESF, através de equipes interdisciplinares, implanta estratégias de atuação, adoção de políticas públicas, atividades comunitárias, organização e planejamento dos serviços de saúde, além da busca ativa, importante ferramenta no combate à doença, interferindo positivamente no acesso e na adesão ao tratamento (MAZZARO CC, et al., 2014).

Diante dessas informações, percebe-se a importância de se conhecer o perfil sociodemográfico do usuário com tuberculose, além de verificar os fatores de agravamento da doença no território e, então, criar estratégias para reverter essa situação. Essas estratégias visam melhorar a capacidade da atenção básica e têm como objetivo reduzir a carga dessa doença e o impacto social e econômico decorrentes de seu contínuo crescimento, logo o objetivo desta pesquisa é identificar a taxa de prevalência de tuberculose pulmonar no território de uma Unidade de Saúde da Família de uma cidade do Pará

\section{MÉTODOS}

Após aprovação pelo Comitê de Ética em Pesquisa (CEP) sob CAAE: 62381116.4.0000.5701, realizouse estudo transversal e observacional descritivo realizada com amostra de 39 participantes com tuberculose pulmonar de ambos os sexos, cadastrados na Estratégia Saúde da Família escolhida na cidade de Belém PA. A coleta de dados foi realizada nos mêses de fevereiro e março de 2018. Todos os participantes assinaram o Termo de Consentimento Livre e Esclarecido (TCLE).

Os dados coletados foram: idade, sexo, raça, grau de escolaridade, atividade remunerada, vinculo empregatício, maior contribuição na renda familiar, quantidade de dependentes da renda, jornada de trabalho, condições legais da residência, quantidade de cômodos, quantidades de pessoas que residem na casa, material da mesma, material do telhado, ventilação da residência, aspectos de saneamento básico e doença crônica, feitos durante o período citado acima. Esses dados foram coletados através de visitas domiciliares, obtendo respostas para o formulário de pesquisa elaborado especificamente para este estudo. Os resultados são apresentados e analisados de forma descritiva e organizados em tabelas. Em seguida, foram compilados no programa Excel 2010, em forma de tabelas para análise quantitativa.

Como critério de inclusão, foi padronizada a inserção de pacientes de ambos os sexos, a partir de 18 anos, que apresentem diagnostico de tuberculose pulmonar, cadastrados na Unidade Saúde da Família Canal escolhida na cidade de Belém/ PA durante o primeiro semestre de 2017, cujos respectivos prontuários estavam devidamente preenchidos e atualizados e que concordaram em colaborar com a pesquisa mediante a assinatura do Termo de Consentimento Livre e Esclarecido.

Como critérios de exclusão, foram excluídos indivíduos pacientes com tuberculose pulmonar a que estiverem fora da faixa etária requerida ou ainda, que não sejam acompanhados pela Estratégia Saúde da Família em questão. Assim como, indivíduos que apresentem omissão de informações em seus prontuários.

\section{RESULTADOS}

Por meio da análise dos dados pesquisados, foi encontrado que a maioria dos pacientes com tuberculose na área escolhida tinha entre $20-30$ anos (29\%) e as demais porcentagens são menor que 20 anos (5\%), entre 31 e 40 anos (10\%), entre $41-50$ anos (22\%), entre 51 e 60 anos (24\%), entre 61 e 70 anos (3\%) e entre 71 e 80 anos (7\%).

Já a distribuição por sexo é dividida em $56 \%$ dos pacientes do sexo feminino e $44 \%$ do masculino. A distribuição por raça é caracterizada com a maioria parda (54\%), depois da raça branca (31\%), negra (15\%). Quando se analisa atividade remunerada, $72 \%$ possui atividade contudo até $68 \%$ desses é de forma autônoma, sem vínculo empregatício. A análise de grau de escolaridade foi avaliada com maioria possuindo ensino fundamental incompleto (44\%), $26 \%$ não é alfabetizado, $20 \%$ possui ensino fundamental completo, $10 \%$ tem ensino fundamental incompleto e nenhum paciente possuia ensino médio completo ou ensino superior. 
Analisando a distribuição dos pacientes com tuberculose segundo contribuição familiar, a maioria relatou a si mesmo como provedor da fonte $(72 \%-n=28)$, depois a mãe como provedora da renda $(20 \%-n=8)$, o companheiro $(8 \%-n=3)$ e nenhum respondeu o pai ou "outro" como provedor da renda (Tabela 1).

Tabela 1 - Distribuição dos pacientes com tuberculose (TB) da Unidade de Saúde da Família segundo maior contribuição para renda familiar em 2018.

\begin{tabular}{lll}
\hline Contribuição para renda & $\mathbf{N}$ & $\%$ \\
\hline Você mesmo & 28 & 72 \\
Cônjuge/Companheiro & 3 & 8 \\
Pai & 0 & 0 \\
Mãe & 8 & 20 \\
Outro & 0 & 0 \\
\hline
\end{tabular}

Fonte: BERNARDO GR, et al., 2020.

A distribuição dos pacientes com tuberculose segundo renda familiar, $91 \%$ relata ter menos que 1 salário mínimo mensal total. Já a análise de quantidade de dependentes da renda familiar, $59 \%$ relatam cinco ou mais e $31 \%$ relataram quatro (Tabela 2 ).

Tabela 2 - Distribuição dos pacientes com tuberculose (TB) da Unidade de Saúde da Família segundo quantidade de dependentes da renda familiar em 2018.

\begin{tabular}{lll}
\hline Dependentes da renda & N & $\%$ \\
\hline Uma & 0 & 0 \\
Duas & 0 & 0 \\
Três & 4 & 10 \\
Quatro & 12 & 31 \\
Cinco ou mais & 23 & 59 \\
\hline
\end{tabular}

Fonte: BERNARDO GR, et al., 2020.

A distribuição dos pacientes segundo jornada de trabalho possui a estatística de $32 \%$ sem jornada fixa, $28 \%$ entre $31-40$ horas semanas, $17 \%$ entre 21 e 30 horas, $15 \%$ entre 11 e 20 horas, $5 \%$ até 10 horas e $3 \%$ mais que 40 horas. Analisando as condições de residência, $49 \%$ relatam que a residência é emprestada ou cedida e $43 \%$ relatam que é alugada.

Analisando a quantidade de cômodos, $41 \%$ relatam três e $39 \%$ relatam quatro. A distribuição dos pacientes segundo material da residência constitui-se que a maioria possui residência de alvenaria $(67 \%)$ e o restante de madeira (33\%), sendo o material do telhado $62 \%$ relatou de telha, $38 \%$ laje de concreto e $5 \%$ de zinco, sendo que $82 \%$ relataram não ter boa ventilação na residência, $8 \%$ com banheiro fora do domicílio, todos com água canalizada e 53\% relataram não ter coleta regular de lixo.

Quando perguntados sobre a presença de doença crônica, 33\% relatou que possuía, sendo que a maioria possui infecção pelo HIV (Vírus da Imunodeficiência Humana) (46\%), 23\% possui asma, 23\% Hipertensão Arterial Sistêmica, 8\% Diabetes Mellitus (Tabela 3).

Tabela 3 - Distribuição dos pacientes com tuberculose (TB) da Unidade de Saúde da Família associados a uma doença crônica em 2018.

\begin{tabular}{lll}
\hline Doença crônica & $\mathbf{N}$ & $\%$ \\
\hline HAS & 3 & 23 \\
Diabetes Mellitus & 1 & 8 \\
HAS e DM & 0 & 0 \\
Asma & 3 & 23 \\
HIV & 6 & 46
\end{tabular}

Fonte: BERNARDO GR, et al., 2020. 


\section{DISCUSSÃO}

Segundo o Programa Nacional de Controle da Tuberculose, entre 2016 e 2017 a TB foi a 4ª causa de mortes por doenças infecciosas no Brasil (SANTA-HELENA ET, et al., 2010; AURELIO M, et al., 2013). A análise dos dados revelou que do total de 39 indivíduos estudados, $29 \%$ se encontra na faixa etária entre 20 e 30 anos. Resultado diferente foi constatado no estudo sobre Perfil dos Pacientes com tuberculose e os fatores relacionados ao abandono do tratamento, o qual revelou que a faixa etária com maior número de pessoas com TB entre 41 a 60 anos (GIROTI SKO, et al., 2010). Essa relação de idade pode impactar diretamente no curso da doença, haja vista a doença em idosos ter mais letalidade pela falta de imunidade e diminuição da atividade basal da respiração e pulmão.

Em relação ao sexo dos pacientes entrevistados 22 (56\%) eram do sexo feminino e 17 (44\%) eram do sexo masculino. Em estudos realizados sobre tuberculose pulmonar abordando o perfil, constatou-se a predominância do sexo masculino, ou seja, estes achados não estão condizentes com a literatura. Inúmeros estudos relatam um predomínio de tuberculose em pacientes do gênero masculino. Ainda contradizendo os dados apresentados, o qual revelou o predomínio do sexo feminino, um estudo sobre perfil e seguimento dos pacientes com tuberculose em município prioritário no Brasil pacientes acompanhados de forma direta apresentaram predomínio do sexo masculino com $65,9 \%$ em relação ao sexo feminino $34,1 \%$ (PEREIRA JC, et al., 2015; BRASIL, 2016).

Segundo a raça, no presente estudo constatou-se que 21 participantes $(54 \%)$ se consideram pardos, representando a maioria da população estudada, seguido de 12 participantes, correspondendo a $31 \%$ que se consideram brancos e 6 (15\%) participantes se consideram negros. Resultado semelhante foi observado no estudo sobre Perfil clínico-epidemiológico de pacientes portadores de tuberculose atendidos em uma unidade municipal de saúde de Belém, Estado do Pará, Brasil, pois revelou predomínio da raça parda, caracterizando $49,02 \%$ de sua amostra (FREITAS WMTM, et al., 2016).

No entanto, as características dos pacientes com tuberculose pulmonar com baciloscopia negativa em uma região com alta prevalência de tuberculose e HIV observou a raça negra em predominância de sua amostra (BRASIL, 2011).

Analisando os dados referentes ao grau de escolaridade, observou-se que 17 (44\%) participantes possuem ensino fundamental incompleto, 10 (26\%) não alfabetizados, 8 (20\%) ensino fundamental completo e $4(10 \%)$ ensino médio incompleto. Não se teve registros de ensino médio completo e com ensino superior completo. Em nenhum caso analisado a profissão requereu um alto grau de instrução, podemos deduzir que nenhum indivíduo possua o terceiro grau de escolaridade. Esse é um achado que pode complicar a epidemiologia da doença, haja vista quem tem mais escolaridade possui geralmente clareza para com o cuidado ou o tratamento correto desta doença. Resultado semelhante foi observado no estudo sobre tuberculose pulmonar, o qual revelou que adultos com menor nível instrucional (analfabetos ou fundamentais incompletos) apresentaram a maior prevalência de pessoas acometidas $(50,4 \%)$ (SILVEIRA MPT, et al., 2007).

Segundo dados acerca da atividade remunerada dos participantes, observou-se que a maioria dos pacientes 28 (72\%) desempenhava alguma função que the oferecia renda mensal e 11 (28\%) não realizam. E se tratando de qual vínculo dessa atividade remunerada 19 (68\%) disseram ser autônomos e 9 (32\%) declararam ter vínculo fixo. Nesse sentido, a renda pessoal ou familiar pode influenciar na doença haja vista quem possui baixa renda geralmente reside em locais que propiciam a maior propagação desta doença (ambientes fechados e sem ventilação).

Resultado similar foi observado no estudo sobre perfil dos pacientes com tuberculose e avaliação do programa nacional de controle da tuberculose em Bagé (RS) o qual mostrou que 123 participantes têm situação laboral semelhante nos dois critérios (SILVEIRA MPT, et al., 2007). Já sobre o a contribuição na renda familiar 28 pessoas, que contabiliza $72 \%$ declararam ser a pessoa que mais contribui, seguindo da mãe com $20 \%$ e o cônjuge com $8 \%$. Outro fator relacionado é a renda mensal familiar onde à maioria, ou seja, $91 \%$ dos pacientes declararam receber menos de um salário mínimo. Ou seja, a maioria dos pacientes 
no estudo apresenta baixa renda, fazendo-nos questionar se pessoas em tal situação estariam mais propensas à infecção, por falta de acesso às informações ou falta de tratamento. Fato constata também em um estudo realizado no Rio grande do Sul (SILVEIRA MPT, et al., 2007).

Ainda relacionado ao perfil econômico, a quantidade de pessoas que dependem dessa renda é de cinco ou mais pessoas, somando 59\%. Nesse contexto, a jornada de trabalho desse paciente tem maior prevalência o item "sem jornada fixa" (32\%), o que demonstra a vulnerabilidade social desse paciente (SILVEIRA MPT, et al., 2007).

Em se tratando de condições legais de moradia, 19 pacientes declararam morar em casa cedida ou emprestada ficando com $49 \%$ do total, 17 (43\%) morar em casa alugada e 3 pessoas (8\%) morar em casa própria. Realidade que se contrapõe a apresentada no estudo tuberculose: perfil epidemiológico do município de Timótio-MG, em que todos os pacientes declararam moradia própria (ASSIS EAR, et al., 2010). Já se tratando da quantidade de cômodos na residência a prevalência ficou com casa de três cômodos com $41 \%$, similar ao estudo Determinação Social da Tuberculose que mostrou o número de cômodos entre 2 e 5 peças entre os entrevistados (LIMAS FM, et al., 2013). Ainda nesse contexto, se tratando de quantas pessoas residem juntas, 12 pacientes (31\%) disseram morar quatro pessoas, 11 (28\%) declararam morar cinco pessoas, 8 (21\%) morar seis pessoas e um empate para os itens "três" e "mais de seis", contendo 3 pacientes para cada.

Em relação ao material que a residência possui, 26 pessoas $(67 \%)$ responderam ser de alvenaria e 13 (33\%) disseram ser de madeira. Resultado semelhante ao encontrado no estudo Determinação Social da Tuberculose o tipo de construção predominante foi o de alvenaria seguido do de madeira (LIMAS FM, et al., 2013). Na mesma linha o material do telhado que predominou na residência dos entrevistados foi telha de barro com $62 \%$, seguido de laje de concreto $38 \%$.

Se tratando da ventilação da residência 32 pacientes (82\%) responderam não ter boa ventilação e 7 pessoas $(18 \%)$ relataram que sim. As condições de moradia devem ser consideradas para uma orientação direcionada e assistência integral do usuário, considerando que existiam pacientes que possuíam casas de apenas três cômodos, com número de pessoas residentes variando entre 4 a 6 pessoas ou mais, sendo o agravo transmitido por via aerógena, os fatores ambientais são importantes, pois quanto maior o tempo que o núcleo seco ficar suspenso no ar, maior será o risco de contágio (LIMAS FM, et al., 2013).

A questão que trata sobre banheiro na residência mostrou $100 \%$ dos pacientes relatando que sim sobre a presença do mesmo, e complementando esse contexto a pergunta feita sobre a localização do mesmo, mostrou que dentro da residência ficou com 36 pessoas que corresponde a $92 \%$ do total ou fora ficando com 3 pessoas correspondendo a $8 \%$. No mesmo segmento, a questão sobre água canalizada na residência mostrou que 39 pacientes, ou seja, 100\% tem sim água canalizada. Além de 23 pacientes (47\%) responderem que tem coleta regular de lixo e 16 (53\%) relatar que não. Realidade que concorda com a apresentada no estudo tuberculose: perfil epidemiológico do município de Timótio-MG, que mostrou que 100\% dos entrevistados tem saneamento básico na área que reside (ASSIS EAR, et al., 2010).

O conhecimento das condições do meio pertinente à saúde, como saneamento básico e moradia são de singular importância no estabelecimento de medidas de promoção da qualidade de vida do assistido, suas famílias e comunidades (SILVEIRA MPT, et al., 2007)

Quando se fala em comorbidades, 26 entrevistados (67\%) responderam não ter presença de doença crônica e 13 pacientes (33\%) afirmaram ter doenças crônicas. Sendo que a de maior prevalência foi HIV com 6 pacientes (46\%), seguida de 3 pacientes com HAS e Asma com 23\% cada uma e Diabetes Mellitus com 1 paciente (8\%). Comparando com um estudo semelhante, encontram-se semelhanças e diferenças, pois observou-se que $18,4 \%$ dos participantes estão classificados como pessoas com HIV e $61,7 \%$ não (SILVEIRA MPT, et al., 2007). Resultado diferente do apresentado foi encontrado em estudo, que afirma que pelo menos uma das doenças crônicas consideradas (diabetes, bronquite ou asma, HAS, HIV) aumentam a chance de contrair tuberculose das pessoas que não referiu nenhuma doença crônica (FRANCO JV, et al., 2010). 
Para a realização desse estudo, enfrentaram-se diversas dificuldades, como a periculosidade da área de abrangência da Unidade Saúde da Família, organização da USF quanto ao armazenamento dos prontuários, mas a maior dificuldade foi encontrar os endereços, pois muitos prontuários não foram preenchidos corretamente, com letra legível ou completo.

\section{CONCLUSÃO}

O presente estudo concluiu que o perfil dos usuários com Tuberculose Pulmonar da Unidade de Saúde da Família é caracterizado em sua maioria por mulheres, cor parda, idade entre 20 e 30 anos, apresentando ensino fundamental incompleto, sem doenças crônicas associadas, entretanto os usuários que apresentam alguma doença crônica associada tem predomínio de HIV; possuem atividade remunerada, porém sem vínculo empregatício e sem jornada de trabalho fixa, apresentando renda menor que 1 salário mínimo; nas moradias tem predomínio de casas cedidas ou emprestadas, de alvenaria, com má ventilação, três cômodos, quatro pessoas residindo na casa, presença de banheiro dentro da residência, com água canalizada e com coleta de lixo regular. Este estudo contribuiu ao encontrar o perfil epidemiológico de pessoas que tratam tubérculos na área a fim de desenvolver a busca ativa e atividades de educação em saúde mais direcionados para a população.

\section{REFERÊNCIAS}

1. AURÉLIO M, et al. Perfil epidemiológico dos pacientes com hipertensão arterial sistêmica por um Programa de Saúde da Família de São Sebastião - DF - Brasil. 2013.

2. ASSIS EAR, et al. Tuberculose: perfil epidemiológico do município de Timóteo-MG, durante o ano de 2007. Rev. Farmácia \& Ciência. 2010; 1: 52-63.

3. BRASIL. Secretária de Estado de Saúde Pública [http://www.saude.pa.gov.br/]. Seminário debate avanços e desafios para o controle da tuberculose no Pará [acesso em 7 nov 2016]. Disponível em: http://www.saude.pa.gov.br/?p=811

4. BRASIL. Secretária de Estado de Saúde Pública [http://www.saude.pa.gov.br/]. Cenário da tuberculose no Pará será tratado em seminário [acesso em 7 nov 2016]. Disponível em: http://www.saude.pa.gov.br/?p=796

5. BRASIL. Programa Nacional de Controle da Tuberculose. Secretaria de Vigilância em Saúde Ministério da Saúde http://portalarquivos.saude.gov.br/images/pdf/2017/fevereiro/21/Apresentacao-sobre-os-principais-indicadores-datuberculose.pd

6. BRASIL. Ministério da Saúde: Programa Nacional de Controle da Tuberculose 2011 [http://portal.saude.gov.br/portal/arquivos/pdf/2site_31_05_2011.pdf].

7. CHAIMOWICZ F. Transição etária da incidência e mortalidade da Tuberculose no Brasil. Rev. Saúde Pública [Internet]. 2001; 35(1): 81-87.

8. BRASIL. Ministério da Saúde. O controle da tuberculose no Brasil: avanços, inovações e desafios. Bolet. Epidem [Internet]. $2013 \mathrm{Fev} ;$ 44(2): 8-9.

9. FRANCO JV, et al. Relação entre a ocorrência de tuberculose e um conjunto de fatores socioeconômicos, demográficos e de saúde da população brasileira usando a PNAD 2003. 19SINAPE. 2010 Jul; 1-6.

10. FREITAS WMTM, et al. Perfil clínico-epidemiológico de pacientes portadores de tuberculose atendidos em uma unidade municipal de saúde de Belém, Estado do Pará, Brasil. Rev Pan-Amaz Saude. 2016 Jun ; 7( 2 ): 45-50.

11. GIROTI SKO, et al. Perfil dos pacientes com tuberculose e os fatores associados ao abandono do trataento. Cogitare Enfermagem. 2010;15(2):271-277.

12. LIMAS FM, et al. Determinação social da tuberculose análise da realidade objetiva do agravo em um serviço de epidemiologia hospitalar. Rev Tempus Actas Saúde Col. 2013 Set; 97-105.

13. MAZZARO CC, et al. Intervenções em Dieta e Pressão Arterial na América Latina - Revisão Sistemática e MetaAnálise. Arq. Bras. Cardiol. [Internet]. 2014; 102(4): 345-354.

14. PEREIRA JC, et al. Profile and follow-up of patients with tuberculosis in a priority city in Brazil. Rev. Saúde Pública. 2015, 27; 49: 6.

15. SANTA-HELENA ET, et al. Fatores associados à não-adesão ao tratamento com anti-hipertensivos em pessoas atendidas em unidades de saúde da família. Cad. Saúde Pública [Internet]. 2010; 26(12): 2389-2398.

16. SILVEIRA MPT, et al. Perfil dos pacientes com tuberculose e avaliação do programa nacional de controle da tuberculose em Bagé (RS). J. bras. pneumol. 2007 Apr; 33(2): 199-205.

17. VENDRAMINI SHF, et al. Tuberculose no idoso: análise do conceito. Rev. Latino-Am. Enfermagem [Internet]. 2003; 11(1): 96-103. 